\title{
Modelling Unsteady Wall Pressures beneath Turbulent Boundary Layers
}

\author{
B-K. Ahn* and W. R. Graham ${ }^{\dagger}$ \\ University of Cambridge, Cambridge, CB2 1PZ, England \\ S. A. Rizzi ${ }^{\ddagger}$ \\ NASA Langley Research Centre, Hampton, VA, 23681-2199, USA
}

\begin{abstract}
As a structural entity of turbulence, hairpin vortices are believed to play a major role in developing and sustaining the turbulence process in the near wall region of turbulent boundary layers and may be regarded as the simplest conceptual model that can account for the essential features of the wall pressure fluctuations. In this work we focus on fully developed typical hairpin vortices and estimate the associated surface pressure distributions and their corresponding spectra. On the basis of the attached eddy model, we develop a representation of the overall surface pressure spectra in terms of the eddy size distribution. Instantaneous wavenumber spectra and spatial correlations are readily derivable from this representation. The model is validated by comparison of predicted wavenumber spectra and cross-correlations with existing empirical models and experimental data.
\end{abstract}

\section{Introduction}

$\mathrm{T}$ HE pressure fluctuations induced by turbulent boundary layers have long been of interest as a significant factor in generating vibration, and hence radiated sound, in many practical applications in which turbulent flow wets a flexible wall. The need to reduce the noise induced by boundary layers implies a requirement for a simple model capable of providing reliable insights into the characteristics of the wall pressure fluctuations.

Turbulent boundary layers exert a shear stress at the wall, and there is a strong connection between this shear stress and the structure of turbulent flows in the vicinity of the wall. Much recent empirical and numerical research has shown that the region of the flow with high vorticity near the wall appears as a bunch of hairpin vortices and longitudinal tubes. Head and Bandyopadhyay ${ }^{1}$ investigated a turbulent boundary layer over a wide range of Reynolds number and observed hairpin vortices which are aligned at $45^{\circ}$ to the wall and convected with a uniform velocity. While these vortices are undergoing a stretching motion, they remain straight and group together to form large scale structures, but they do not appear to be interacting strongly with each other. In flow visualization studies, Acarlar and Smith ${ }^{2}$ confirmed this feature of the hairpin vortices.

We therefore focus attention on fully developed hairpin vortices inclined at an angle of $45^{\circ}$ to the wall and estimate the associated surface pressure distributions and their corresponding spectra. On the assumption that fully developed hairpin vortices are governed by inviscid dynamics, we obtain an exact formulation for the stagnation pressure, in terms of a Green function integral along the vortex lines. We then evaluate the

${ }^{*}$ Research Student, Department of Engineering, Trumpington Street, Cambridge, AIAA Student Member.

$\dagger$ Faculty, Department of Engineering, Trumpington Street, Cambridge.

$\ddagger$ Aerospace Engineer, Structural Acoustics Branch, 2 North Dryden Street, Hampton VA, AIAA Associate Fellow.

$$
1 \text { of } 12
$$

American Institute of Aeronautics and Astronautics 
surface static pressure by subtracting the dynamic pressure from the results of this formulation applied to our vortex geometry.

On the basis of the attached eddy model,$^{3,4}$ which implies that the form of the wave number spectrum can be deduced from the properties of a single eddy, we develop the expressions needed for the surface pressure spectra in terms of eddy number-density. This approach draws on flow visualization evidence, which indicates that the number of eddies observed in both streamwise and spanwise directions of the flow is inversely proportional to their size. The overall wave number spectrum consists of contributions from eddies of all sizes, weighted by the number-density. We then validate our model against existing descriptions and measurements of turbulent boundary layer pressures. We examine the predicted wave number spectra and compare them with those of the Corcos and Efimtsov model ${ }^{5,6}$ and also with the results of flight test measurements carried out by NASA, Boeing and ANTK Tupolev on the Tu-144LL supersonic aircraft. ${ }^{7}$

\section{Relationship between turbulent structures and wall pressures}

Many analytical approaches linking velocity and wall pressure fluctuations in turbulent boundary layers have been proposed. One of them, for incompressible fluctuations, begins with the Poisson equation $^{8,9}$

$$
\nabla^{2} p=-\rho \frac{\partial^{2}\left(u_{i} u_{j}\right)}{\partial x_{i} \partial x_{j}}
$$

The dominant contribution to the wall pressure fluctuations in turbulent boundary layers comes from Reynolds stresses, and the pressure field induced by turbulent flows is obtained by solving this equation. However, Reynolds stresses cannot be calculated explicitly until the complete turbulent flow is known. The traditional approach consists of determining models for Reynolds stresses based on empirical statistics so that the Navier Stokes equation can be closed. The characteristics of the wall pressure fluctuations have been identified through spectral analysis. Significant contributions to the high

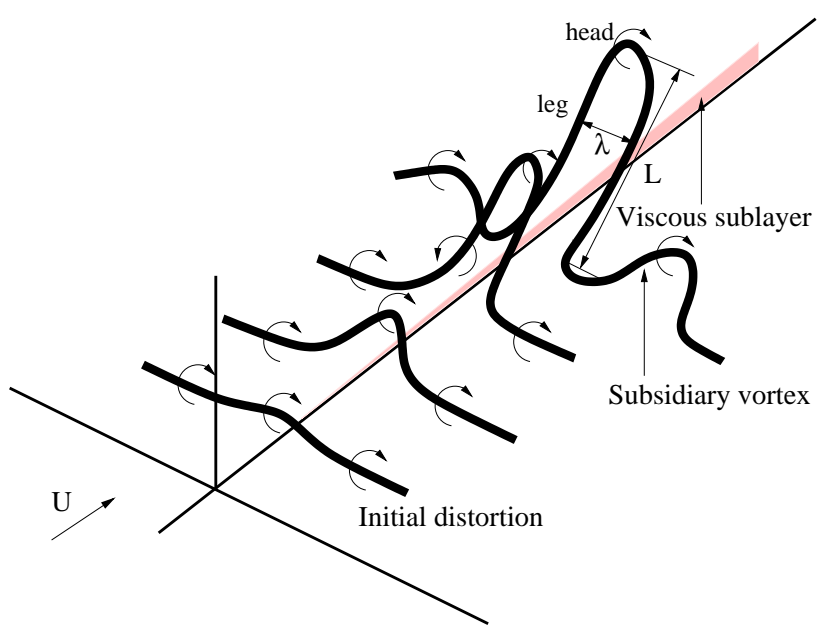

Figure 1. Schematic view of the typical hairpin vortex's generation. wave number pressure components are attributed to activity in the near wall region, while contributions to the low wave number components are associated with larger structures in the outer layers.

The long-time evolution of the vorticity field above the wall is probably not deterministic in the usual sense and may be regarded as chaotic. Nevertheless, it is clear ${ }^{10}$ that vortices are a dominant feature of the instantaneous turbulent flow, and furthermore that some events associated with vortices are observed to occur repeatedly. Increasingly, the importance of the coherent structures of turbulent boundary layers and their contributions to the wall pressure fluctuations is being recognized. These features help in identifying characteristics of the surface pressures and their spectra in forming a model for the pressure field beneath turbulent boundary layers. Hence a better understanding of the surface pressures induced by typical turbulent structures is required.

Figure 1 presents a schematic view of the typical hairpin vortex's generation. ${ }^{10,11}$ The initial disturbance starts to grow and generate a shape in streamwise direction. Rapidly a vortex head develops, rises from the wall, and bends back in the shear flow. However there is no clear explanation for its $45^{\circ}$ orientation, other than the fact that the maximum mean rate of strain is at $45^{\circ}$. As time increases, the vortex head moves farther from the wall while the legs move progressively closer to the surface, and the streamwise structures give way to hairpin vortices until the wake region where the structures are predominantly hairpin-

\section{2 of 12}

American Institute of Aeronautics and Astronautics 
like. The original disturbance spreads laterally and produces subsidiary vortices. The development of the subsidiary vortices allows the original disturbance to multiply and spread in the spanwise direction through an interaction with the background shear flow. The distance between the vortex legs $(\lambda)$ depends on the level of the background shear flow.

\section{Numerical formulation}

Here we derive the equations for determining the surface pressure field induced by the hairpin vortex, which is regarded as two symmetric thin vortex lines inclined at $45^{\circ}$ to the wall joined by a horizontal head (see Figure 2). In order to satisfies the wall boundary condition image vortex lines are placed symmetrically beneath them. A suitable cut-off distance $(c)$ is adopted to avoid the singularity in the self-induction integral. ${ }^{12}$ Smith and Metzler ${ }^{13}$ have suggested that the typical vortex has a core diameter of about $30 \nu / u_{\tau}$ and the spacing of the vortex pair is $100 \nu / u_{\tau}$ over a range of Reynolds numbers in the near-wall regions. $\mathrm{Crow}^{12}$ has shown the relation between the cut-off distance $(c)$ and vortex core diameter $(d)$ is $c / d \simeq 1 / 3$. The cut-off distance is thus taken as proportional to the vortex core diameter which is also proportional to the distance between the two legs.

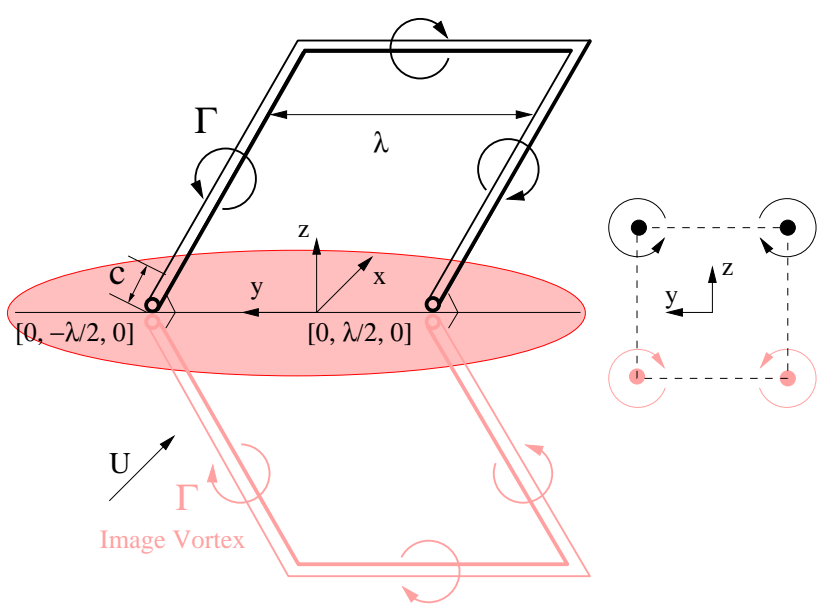

We follow the numerical formulation derived by Dhanak and Dowling ${ }^{14}$ for the surface pressure induced due to a developing horse-shoe shaped vortex. By using the vector identity, $(\underline{u} \cdot \nabla) \underline{u}=\nabla\left(\frac{1}{2} \underline{u} \cdot \underline{u}\right)-\underline{u} \times \underline{\omega}$, and introducing the stagnation pressure, $p(\underline{x}, t)=$ $p^{\prime}+\frac{1}{2} \rho|\underline{u}|^{2}$, the momentum equation may be written in the form

$$
\rho\left(\frac{\partial \underline{u}}{\partial t}+\underline{\omega} \times \underline{u}\right)=-\nabla p+\mu \nabla^{2} \underline{u}
$$

The normal component of the momentum equation should satisfy the rigid wall boundary condition:

$$
\left.\frac{\partial p}{\partial z}\right|_{z=0}=-\rho(\underline{\omega} \times \underline{u})_{z}+\mu \nabla^{2} u_{z} \simeq 0
$$

Taking the divergence of each term in Eq. (2) and using the continuity equation $(\nabla \cdot \underline{u}=0)$ for an incompressible flow, a Poisson equation for the stagnation pressure can be obtained:

$$
\nabla^{2} p=-\rho \nabla \cdot(\underline{\omega} \times \underline{u})
$$

The solution of the Poisson equation can be determined in terms of the Green function

$$
G(\underline{\xi} \mid \underline{x})=-\frac{1}{4 \pi|\underline{\xi}-\underline{x}|}-\frac{1}{4 \pi\left|\underline{\xi}-\underline{x^{\prime}}\right|}=-\frac{1}{4 \pi}\left(\frac{1}{r}+\frac{1}{r^{\prime}}\right)
$$

where $\underline{x}^{\prime}$ is the image point of $\underline{x}$, and $G(\underline{\xi} \mid \underline{x})$ satisfies

$$
\nabla_{\xi}^{2} G=\delta(\underline{\xi}-\underline{x})
$$

$$
3 \text { of } 12
$$


with $\frac{\partial G}{\partial \xi_{3}}=0$ on $\xi_{3}=0$ and $G(|\underline{\xi}-\underline{x}|) \rightarrow 0$ as $|\underline{\xi}| \rightarrow \infty$. By using Green's theorem and these boundary conditions, the stagnation pressure is written in terms of a volume integral over the region in which the vorticity is nonzero, and a surface integral due to viscous effects.

$$
p(\underline{x})=\int_{V} \nabla_{\xi} G(\underline{\xi} \mid \underline{x}) \cdot \rho(\underline{\omega} \times \underline{u}) d^{3} \xi+\int_{S} \mu G \nabla^{2} u_{3} d \xi_{1} d \xi_{2}
$$

If one assumes that the fully developed hairpin vortex is governed by inviscid dynamics, then the equation for the stagnation pressure may be simplified further, such that without viscous terms:

$$
p(\underline{x})=\rho \int_{V} \nabla_{\xi} G(\underline{\xi} \mid \underline{x}) \cdot(\underline{\omega} \times \underline{u}) d^{3} \xi
$$

\section{Application of the attached eddy model to the surface pressure spectra}

In experimental and theoretical works, Perry et $\mathrm{al}^{3,4}$ showed that an isolated hairpin vortex undergoes a stretching motion in which the vortex height $L$ increases uniformly with time and the distance $\lambda$ between the two legs of the hairpin vortex at the wall decreases such that the product $\lambda L$ remains constant. They also showed a geometrically similar random array of hairpin vortices exists in the turbulent wall region and all eddy arrays have the same velocity scale $\left(\sim u_{\tau}\right)$. They applied the observation that the only velocity scale in the log-law region
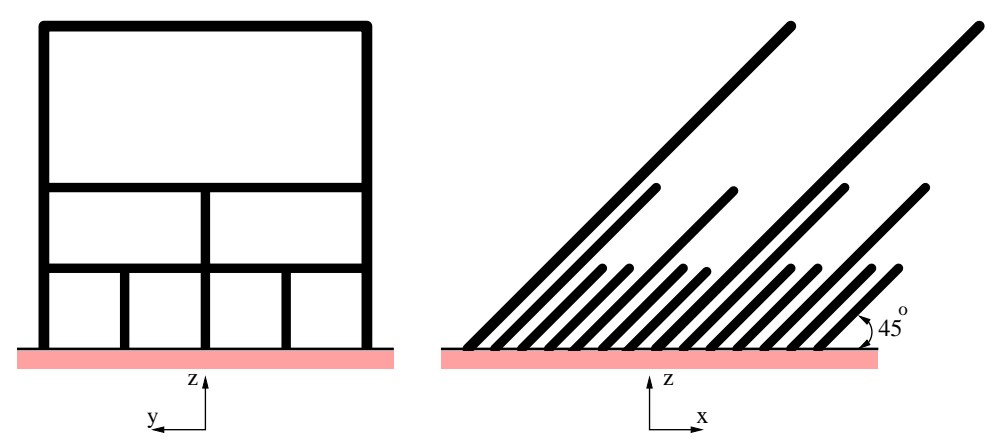

Figure 3. Schematic representation of eddy size distribution in spanwise and streamwise directions. is $u_{\tau}$, the friction velocity, to each individual eddy. Thus the typical hairpin vortex, with length scale $\lambda$, has vorticity $\omega \sim u_{\tau} \lambda^{-1}$ and circulation $\Gamma \sim u_{\tau} \lambda$.

Flow visualisation work that they cite indicates that the number of eddies observed in both streamwise (x) and spanwise (y) directions decreases like $\lambda^{-1}$ (see Figure 3). This implies that the number of eddies per unit area around a scale $\lambda$ should be four times that around the scale $2 \lambda$, and this requirement is fulfilled by the eddy number density $n(\lambda)=N \lambda^{-3}$, with $\mathrm{N}$ constant. The physical interpretation of the increase in circulation with size is linked to the $\lambda^{-1}$ distribution of eddies in the streamwise direction, which implies combination as they evolve and grow.

The key point in deriving spectra from the attached eddy model is that the eddies are uncorrelated (on average). This implies that the form of a spectrum can be deduced from the properties of a single eddy. This approach is applied to velocity auto and cross-spectra by Perry et al, but it can be equally well used for pressure spectra. Here, we develop an alternative approach, in terms of eddy number density, to obtain expressions needed for the surface pressure spectra. The spectrum is defined by

$$
S\left(k_{x}, k_{y}\right)=\int_{-\infty}^{\infty} \int_{-\infty}^{\infty} R(\xi, \eta) e^{-i \xi k_{x}} e^{-i \eta k_{y}} d \xi d \eta
$$

with the ensemble average of the surface $\operatorname{pressure}(p)$,

$$
R(\xi, \eta)=\overline{p(x, y) p(x+\xi, y+\eta)}
$$

It can be related to the Fourier transform of the pressure field,

$$
\begin{gathered}
P\left(k_{x}, k_{y}\right)=\int_{-\infty}^{\infty} \int_{-\infty}^{\infty} p(x, y) e^{-i k_{x}} e^{-i k_{y}} d x d y \\
4 \text { of } 12
\end{gathered}
$$


via generalized function theory, with the result that

$$
(2 \pi)^{2} \delta\left(k_{x}+\dot{k}_{x}\right) \delta\left(k_{y}+\dot{k}_{y}\right) S\left(k_{x}, k_{y}\right)=\overline{P\left(k_{x}, k_{y}\right) P\left(\dot{k}_{x}, \dot{k}_{y}\right)}
$$

We write the pressure field arising from all eddies,

$$
p(x, y)=\sum_{N_{e}} p_{e}\left(x-x_{e}, y-y_{e}, \lambda\right)
$$

where $\left(x_{e}, y_{e}\right)$ is the eddy location. The Fourier transform is thus

$$
P\left(k_{x}, k_{y}\right)=\sum_{N_{e}} P_{e}\left(k_{x}, k_{y}, \lambda\right) e^{-i k_{x} x_{e}} e^{-i k_{y} y_{e}}
$$

where $N_{e}$ is the number of eddies and $P_{e}\left(k_{x}, k_{y}, \lambda\right)$ is the Fourier transform of the pressure field due to an individual eddy, and Eq. (12) becomes

$$
(2 \pi)^{2} \delta\left(k_{x}+\dot{k}_{x}\right) \delta\left(k_{y}+\dot{k}_{y}\right) S\left(k_{x}, k_{y}\right)=\sum_{N_{e}} P_{e}\left(k_{x}, k_{y}, \lambda\right) P_{e}\left(\dot{k}_{x}, \dot{k}_{y} \lambda\right) e^{-i\left(k_{x}+\dot{k}_{x}\right) x_{e}} e^{-i\left(k_{y}+\dot{k}_{y}\right) y_{e}}
$$

Note that the ensemble average has removed all terms involving contributions from different eddies. Then the summation is replaced with an integral over eddy location and scale, giving

$$
\begin{gathered}
(2 \pi)^{2} \delta\left(k_{x}+\dot{k}_{x}\right) \delta\left(k_{y}+\dot{k}_{y}\right) S\left(k_{x}, k_{y}\right) \\
=\int_{\lambda_{m}}^{\delta} \iint_{-\infty}^{\infty} n(\lambda) P_{e}\left(k_{x}, k_{y}, \lambda\right) P_{e}\left(\dot{k}_{x}, \dot{k}_{y} \lambda\right) e^{-i\left(k_{x}+\dot{k}_{x}\right) x_{e}} e^{-i\left(k_{y}+\dot{k}_{y}\right) y_{e}} d x_{e} d y_{e} d \lambda
\end{gathered}
$$

Here $\lambda_{m}$ is the scale of the smallest eddy (around $100 \nu / u_{\tau}$ ), and $\delta$ the boundary layer thickness which is the scale of the largest eddy. Integration over $\dot{k}_{x}$ and $\dot{k}_{y}$ now yields

$$
S\left(k_{x}, k_{y}\right)=\int_{\lambda_{m}}^{\delta} n(\lambda)\left|P_{e}\left(k_{x}, k_{y}, \lambda\right)\right|^{2} d \lambda
$$

In the same way, the streamwise and spanwise spectrum are respectively expressed as follow:

$$
\begin{aligned}
& S\left(k_{x}, y\right)=\int_{\lambda_{m}}^{\delta} \int_{-\infty}^{\infty} n(\lambda)\left|P_{e}\left(k_{x}, y, \lambda\right)\right|^{2} d y d \lambda \\
& S\left(x, k_{y}\right)=\int_{\lambda_{m}}^{\delta} \int_{-\infty}^{\infty} n(\lambda)\left|P_{e}\left(x, k_{y}, \lambda\right)\right|^{2} d x d \lambda
\end{aligned}
$$

\section{Non-dimensionalisation and the 'universal' spectrum}

The surface pressure due to the eddy is a function of the fluid density $\rho$, the vortex strength $\Gamma$, the eddy core size $d$, the length scale $\lambda$, and the location $x, y$; that is,

$$
p_{e}=f(\rho, \Gamma, d, \lambda, x, y)
$$

We investigated the dependence of the pressure field on the eddy core size and found that it is weak. The non-dimensional surface pressure may then be expressed as

$$
p_{e}(\rho, \Gamma, \lambda, x, y)=\frac{1}{2} \rho\left(\frac{\Gamma}{\lambda}\right)^{2} \tilde{p_{e}}(x / \lambda, y / \lambda)
$$

$$
5 \text { of } 12
$$


As only the variation in $x, y$ and $\lambda$ is of interest, the dependence on other variables will be suppressed. The nondimensional streamwise wavenumber spectrum is

$$
P_{e}\left(k_{x}, k_{y}, \lambda\right)=\frac{1}{2} \rho \frac{\Gamma^{2}}{\lambda} \tilde{P}_{e}\left(\tilde{k}_{x}, \tilde{k}_{y}\right)
$$

where $\tilde{k}_{x}=\lambda k_{x}, \tilde{k}_{y}=\lambda k_{y}, \tilde{x}=x / \lambda, \tilde{y}=y / \lambda$ and

$$
P_{e}\left(\tilde{k}_{x}, \tilde{k}_{y}\right)=\int_{-\infty}^{\infty} \int_{-\infty}^{\infty} \tilde{P}_{e}(\tilde{x}, \tilde{y}) e^{-i \tilde{k}_{x} \tilde{x}} e^{-i \tilde{k}_{y} \tilde{y}} d \tilde{x} d \tilde{y} .
$$

Substitution into Eq. (17) with the scaling $\Gamma \sim u_{\tau} \lambda$ yields

$$
S\left(\tilde{k}_{x}, \tilde{k}_{y}\right)=\left(\frac{1}{2} \rho u_{\tau}^{2}\right)^{2} \int_{\lambda_{m}}^{\delta} N_{e} \lambda\left|\tilde{P}_{e}\left(\tilde{k}_{x}, \tilde{k}_{y}\right)\right|^{2} d \lambda
$$

Finally, one usually wishes to consider the spectrum in non-dimensionalised form. Equation (24) can be manipulated to provide the required expression:

$$
\frac{S\left(\hat{k}_{x}, \hat{k}_{y}\right)}{\left(\frac{1}{2} \rho u_{\tau}^{2}\right)^{2} \delta^{2}}=\int_{\hat{\lambda}_{m}}^{1} N_{e} \hat{\lambda}\left|\tilde{P}_{e}\left(\hat{\lambda} \hat{k}_{x}, \hat{\lambda} \tilde{k}_{y}\right)\right|^{2} d \hat{\lambda}
$$

where $\hat{k}_{x}=\delta k_{x}, \hat{k}_{y}=\delta k_{y}$ and $\hat{\lambda}=\lambda / \delta$. Firstly we evaluate the 'universal' spectrum

$$
S\left(\lambda k_{x}, \lambda k_{y}\right)=\left|\tilde{P}_{e}\left(\lambda k_{x}, \lambda k_{y}\right)\right|^{2}
$$

for a range of values of the arguments $\lambda k_{x}, \lambda k_{y}$, and store this in a look-up table. By using a suitable quadrature, we then evaluate Eq. (25). Here, whenever the integrand is required for a given value of $\hat{\lambda}$, we have $\lambda k=\hat{\lambda} \hat{k}$, hence defining the argument of the 'universal' spectrum and therefore the appropriate look-up table location. With the same procedure, the non-dimensional streamwise and spanwise wavenumber spectrum are respectively expressed as follows:

$$
\begin{aligned}
& \frac{S\left(\hat{k}_{x}\right)}{\left(\frac{1}{2} \rho u_{\tau}^{2}\right)^{2} \delta}=\int_{\hat{\lambda}_{m}}^{1} \int_{-\infty}^{\infty} N_{e}\left|\tilde{P}_{e}\left(\hat{\lambda} \hat{k}_{x}, \tilde{y}\right)\right|^{2} d \tilde{y} d \hat{\lambda} \\
& \frac{S\left(\hat{k}_{y}\right)}{\left(\frac{1}{2} \rho u_{\tau}^{2}\right)^{2} \delta}=\int_{\hat{\lambda}_{m}}^{1} \int_{-\infty}^{\infty} N_{e}\left|\tilde{P}_{e}\left(\tilde{x}, \hat{\lambda} \hat{k}_{y}\right)\right|^{2} d \tilde{x} d \hat{\lambda}
\end{aligned}
$$

In these cases, the 'universal' spectra are respectively defined by

$$
S\left(\lambda k_{x}\right)=\int_{-\infty}^{\infty}\left|\tilde{P}_{e}\left(\lambda k_{x}, \tilde{y}\right)\right|^{2} d \tilde{y}
$$

and

$$
S\left(\lambda k_{y}\right)=\int_{-\infty}^{\infty}\left|\tilde{P}_{e}\left(\tilde{x}, \lambda k_{y}\right)\right|^{2} d \tilde{x}
$$

\section{Wavenumber spectrum model}

On the basis of the Corcos ${ }^{5}$ and Efimtsov models,${ }^{6}$ we derive an expression for the wavenumber spectrum, evaluate its value and compare with our results. The Corcos model for the spatial correlation between two pressures separated by $(\xi, \eta)$ in $(x, y)$ is

$$
\begin{gathered}
S(\xi, \eta, \omega)=\Phi(\omega) e^{\left[-\alpha_{x}|\omega \xi| / U_{c}-\alpha_{\eta}|\omega \eta| / U_{c}+i \omega \xi / U_{c}\right]} \\
6 \text { of } 12
\end{gathered}
$$


where $\alpha_{x}$ and $\alpha_{y}$ are parameters chosen to yield the best agreement with experiment and $\Phi(\omega)$ is the point pressure spectrum. The Efimtsov model considers the dependence of spatial correlation on the boundary layer thickness $(\delta)$, as well as the streamwise $(\xi)$ and spanwise $(\eta)$ spatial separation and it is obtained by replacing $\alpha_{x}$ and $\alpha_{y}$ in Eq. (31) by $U_{c} /|\omega| \Lambda_{x}$ and $U_{c} /|\omega| \Lambda_{y}$ respectively. The correlation scales $\Lambda_{x}$ and $\Lambda_{y}$ are

$$
\begin{aligned}
\frac{\Lambda_{x}}{\delta} & =\left[\left(\frac{a_{1} S h}{U_{c} / U_{\tau}}\right)^{2}+\frac{a_{2}^{2}}{S h^{2}+\left(a_{2} / a_{3}\right)^{2}}\right]^{-1 / 2} \\
\frac{\Lambda_{y}}{\delta} & =\left[\left(\frac{a_{4} S h}{U_{c} / U_{\tau}}\right)^{2}+a_{7}^{2}\right]^{-1 / 2} \text { for } M \geq 0.9 \\
& =\left[\left(\frac{a_{4} S h}{U_{c} / U_{\tau}}\right)^{2}+\frac{a_{5}^{2}}{S h^{2}+\left(a_{5} / a_{6}\right)^{2}}\right]^{-1 / 2} \text { for } M \leq 0.75,
\end{aligned}
$$

where $M$ is the Mach number, and $S h=\omega \delta / u_{\tau}$. The average values of the empirical constants, $a_{1 \sim 7}$, are $0.1,0.728,1.54,0.77,548$ and 13.5 respectively. We seek to relate the streamwise and spanwise wavenumber spectrum to the frequency spectrum:

$$
\begin{aligned}
& S\left(k_{x}\right)=\frac{1}{2 \pi} \int_{-\infty}^{\infty} \int_{-\infty}^{\infty} S(\xi, 0, \omega) e^{-i k_{x} \xi} d \xi d \omega \\
& S\left(k_{y}\right)=\frac{1}{2 \pi} \int_{-\infty}^{\infty} \int_{-\infty}^{\infty} S(0, \eta, \omega) e^{-i k_{y} \eta} d \eta d \omega
\end{aligned}
$$

The Corcos and Efimtsov models give:

$$
\begin{aligned}
S\left(k_{x}\right) & =\frac{1}{\pi} \int_{-\infty}^{\infty} \Phi(\omega) \frac{\Lambda_{x}}{1+\Lambda_{x}^{2}\left(k_{x}-\omega / U_{c}\right)^{2}} d \omega \\
S\left(k_{y}\right) & =\frac{1}{\pi} \int_{-\infty}^{\infty} \Phi(\omega) \frac{\Lambda_{y}}{1+\left(k_{y} \Lambda_{y}\right)^{2}} d \omega
\end{aligned}
$$

Efimtsov $^{6}$ gives the frequency spectrum as a function of the Strouhal number $(S h)$, which was approximated by the flight-test results,

$$
\Phi(\omega)=\left(\frac{\tau^{2} \delta}{u_{\tau}}\right) * 0.01 \pi\left[1+0.02(S h)^{2 / 3}\right]^{-1}
$$

and the relation $p_{r m s}=3.4 \tau^{9}$ then implies an upper frequency limit corresponding to $S h=3980$ in Eq. (36) and (37).

\section{Results and Discussions}

Figure 4 on page 10 illustrates the surface pressure contours of $\tilde{p}_{e}$ in Eq. (21) due to the different eddy core sizes, and it shows that the dependence of the pressure field on the eddy core size is sufficiently weak that the non-dimensionalised surface pressure can be represented without its contribution. In figure 5 on page 11 streamwise and spanwise pressure spectra and their corresponding universal spectra are shown. On the basis of the attached eddy model, the streamwise and spanwise wavenumber spectrum for the whole scale of eddies are evaluated and compared with the Efimtsov model in Figure 6 on page 12. There have been arguments for the spectrum shape, -1 and -5 slope,${ }^{9,15}$ but its physical explanation is not yet clear. Perry et $\mathrm{al}^{4}$ suggest that there is a range of wavenumbers where the streamwise velocity spectrum $\Phi(k)$ at a given height $z$ depends on the mean wall shear velocity $\left(u_{\tau}\right)$, wave number $(k)$, eddy scale $(\delta)$ and height $(z)$, but not viscosity. Furthermore, at the low end of this range, the velocity fluctuations are associated with eddies of scale much greater than $\mathrm{z}$, and so

$$
\Phi(k)=\delta u_{\tau}^{2} g_{1}(k \delta)
$$

$$
7 \text { of } 12
$$


where $g_{1}$ is a dimensionless function. Conversely, at the higher end of the range one would expect eddies of scale $O(z)$ to contribute, and that this contribution should be independent of $\delta$. This implies

$$
\Phi(k)=z u_{\tau}^{2} g_{2}(k z)
$$

If we now postulate an 'overlap region' where both of these forms hold, then

$$
\frac{g_{1}(k \delta)}{g_{2}(k z)}=\frac{z}{\delta}
$$

implying that $g_{1}(k \delta) \sim(k \delta)^{-1}, g_{2}(k z) \sim(k z)^{-1}$, and hence that $\Phi(k) \sim k^{-1}$ in this region. Perry et al's experiment supports this feature. It is hard to see how these arguments could be applied directly to the wall pressures, as there is no height variable $z$ present. However, the other length scale on which the wavenumber spectrum clearly depends is $\lambda_{m}$, the smallest eddy scale, and one could perhaps argue for dependencies of the form

$$
S(k)=\left(1 / 2 \rho u_{\tau}^{2}\right)^{2} \delta g_{1}(k \delta)
$$

and

$$
S(k)=\left(1 / 2 \rho u_{\tau}^{2}\right)^{2} \lambda_{m} g_{2}\left(k \lambda_{m}\right)
$$

at low and high wavenumbers respectively, in which case any overlap region would have -l slope. In any case, the current model clearly predicts a region of -1 slope in both spectra, whereas this is absent from the streamwise Efimtsov spectrum. None of the spectra exhibit a distinct region of -5 slope. Figure 7 (a) on page 12 shows the spatial correlations obtained by inverse Fourier transform compared with the results of flight test measurements carried out by NASA, Boeing and ANTK Tupolev on the Tu-144LL supersonic aircraft. ${ }^{7}$ Here the spatial separations are normalised by different boundary layer thicknesses: the one marked 'US' is used by Boeing $(\delta=0.49 \mathrm{~m})$ and the others marked 'Russia' by the Tupolev $(\delta=0.30 \mathrm{~m})$ for the same experimental conditions (distance from aircraft nose $=49.3 \mathrm{~m}, \mathrm{M}=0.74$ ). The predicted correlations agree well with the Tupolev result. Finally, 2-dimensional universal spectrum is provided in Figure 7 (b) on page 12.

\section{Conclusion}

The present hairpin vortex model shows that it is capable of predicting the properties of the wall pressure field, and is therefore a promising candidate for use in exploring features less readily obtainable by other methods, eg off-axis correlations. Additional comparisons of streamwise and spanwise wavenumber spectra and correlations are presently being made to improve the model. Use of the model for oblique pressure correlations will also be investigated.

\section{References} 337.

${ }^{1}$ Head, M. R. and Bandyopadhyay, P., "New aspects of turbulent boundary layer structure," JFM, Vol. 107, 1981, pp. 297-

${ }^{2}$ Acarlar, M. S. and Smith, C. R., "A study of hairpin vortices in a laminar boundary layer," JFM, Vol. 175, 1987, pp. $1-41$.

${ }^{3}$ Perry, A. E. and Chong, S., "On the mechanism of wall turbulence," JFM, Vol. 119, 1982, pp. 173-217.

${ }^{4}$ Perry, A. E., Henbest, S., and Chong, M. S., "A theoretical and experimental study of wall turbulence," JFM, Vol. 165, 1986, pp. 163-199. 378-70.

${ }^{5}$ Corcos, G. M., "The structure of the turbulent pressure field in boudary-layer flows," JFM, Vol. 18(3), 1964, pp. 353-

${ }^{6}$ Efimtsov, B. M., "Characteristics of the field of turbulent wall pressure fluctuations at large Reynolds numbers," Soviet Physics-Acoustics, Vol. 28, 1982, pp. 289-292.

${ }^{7}$ Rizzi, S. A., Rackl, R. G., and Andrianov, E. V., "Flight Test Measurements From The Tu-144LL Structure/Cabin Noise Experiment," Report NASA/TM-2000-209858, NASA, Langley Research Center, 2000.

\section{8 of 12}

American Institute of Aeronautics and Astronautics 
${ }^{8}$ Kraichnan, R. H., "Pressure fluctuations in turbulent flow over a flat plate," JASA, Vol. 28, 1956, pp. 379-390.

${ }^{9}$ Blake, W. K., Mechanics of flow-induced sound and vibration, Volume II, Academic Press, 1986.

${ }^{10}$ Smith, C. R. and Walker, J. D. A., "On the dynamics of near-wall turbulence," Philosophical Transactions of The Royal Society of London, Vol. 336, 1991, pp. 131-172.

${ }^{11}$ Townsend, A. A., The structure of turbulent shear flow, Cambridge University Press, 1976.

${ }^{12}$ Crow, S. C., "Stability theory for a pair of trailing vortices," Report D1-82-0918, Flight Science Laboratory, Boeing Scientific Research Laboratories, 1969.

${ }^{13}$ Smith, C. R. and Metzler, S. P., "The characteristics of low-speed streaks in the near-wall region of a turbulent boundary layer," JFM, Vol. 129, 1983, pp. 27-54.

${ }^{14}$ Dhanak, M. R. and Dowling, A. P., "On the pressure fluctuations induced by coherent vortex motion near a surface," 26th AIAA Fluid Dynamics Conference, 1995, pp. 1-10.

${ }^{15}$ Bradshaw, P., "Inactive motion and pressure fluctuations in turbulent boundary layers," JFM, Vol. 30, 1967, pp. 241-258. 


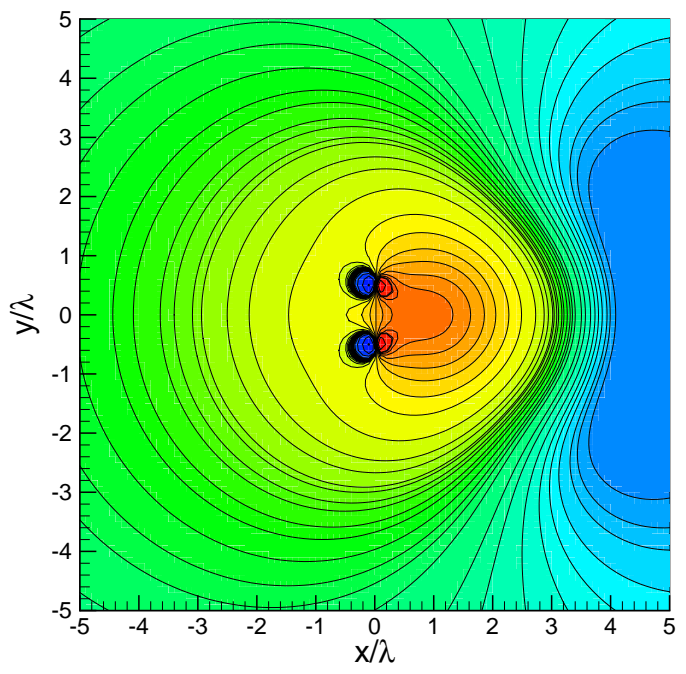

(a)

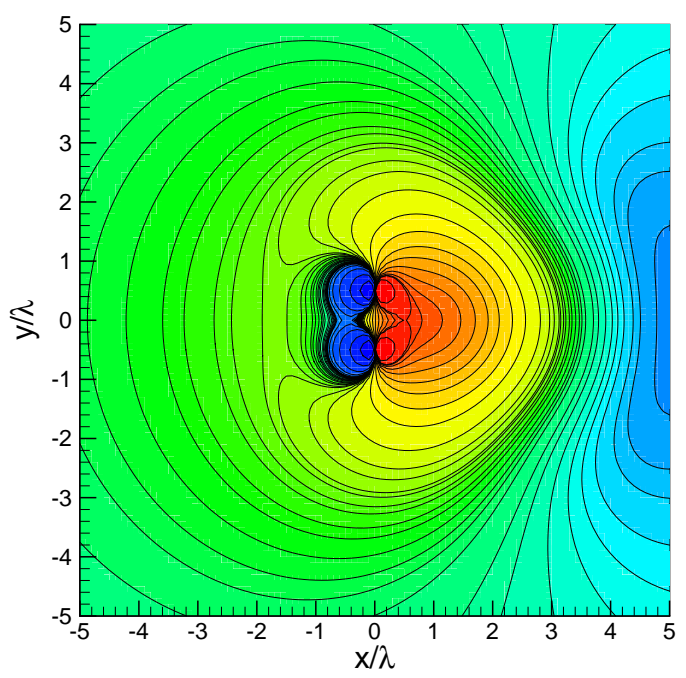

(c)

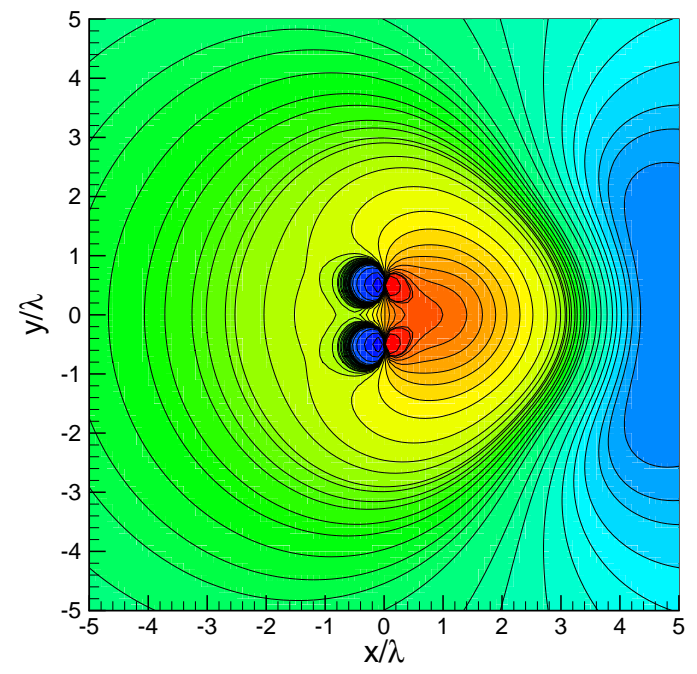

(b)

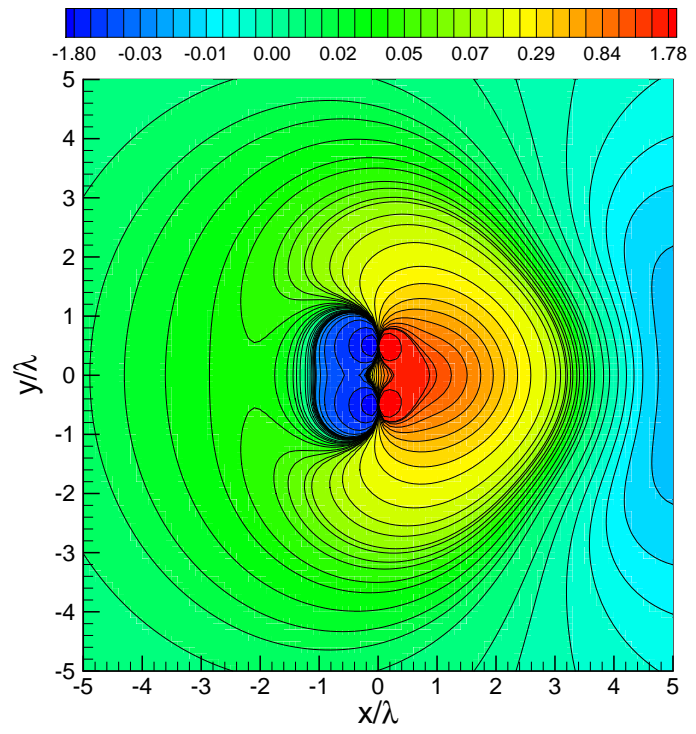

(d)

Figure 4. Surface pressure contours according to different eddy core sizes: (a) $\mathbf{d}=10^{4} \frac{\nu}{u_{\tau}},(\mathbf{b}) \mathbf{d}=10^{3} \frac{\nu}{u_{\tau}},(\mathbf{c})$ $\mathbf{d}=10^{2} \frac{\nu}{u_{\tau}}$ and (d) $\mathbf{d}=10 \frac{\nu}{u_{\tau}}$. 


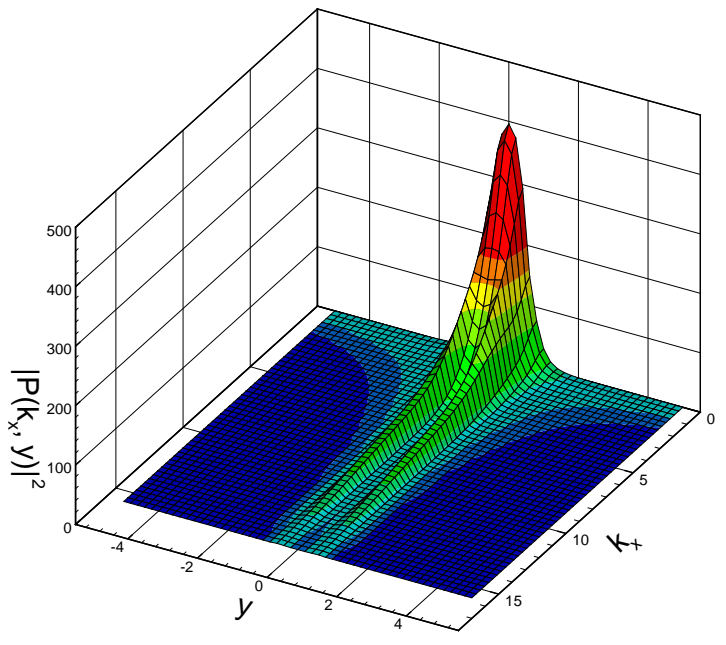

(a)

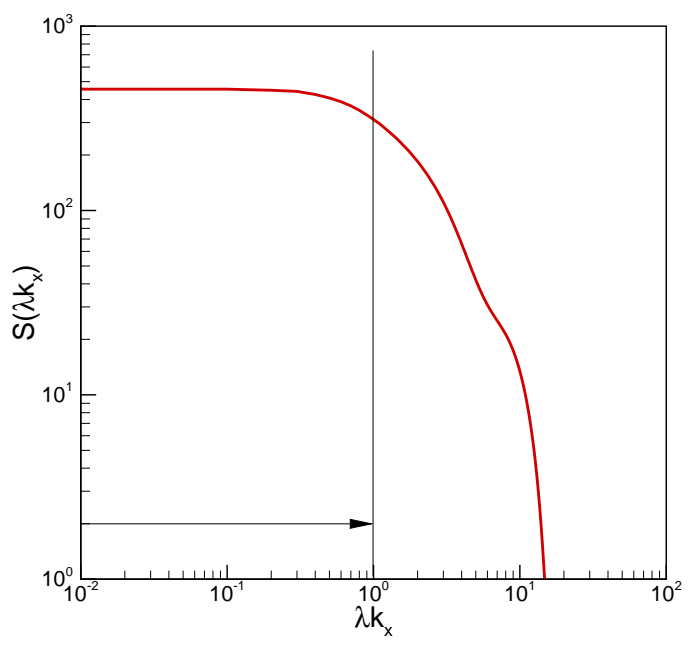

(c)

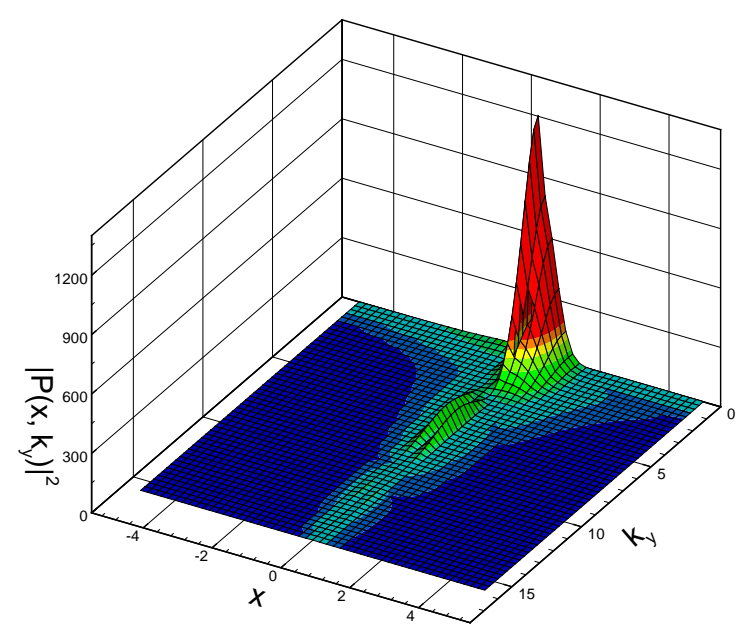

(b)

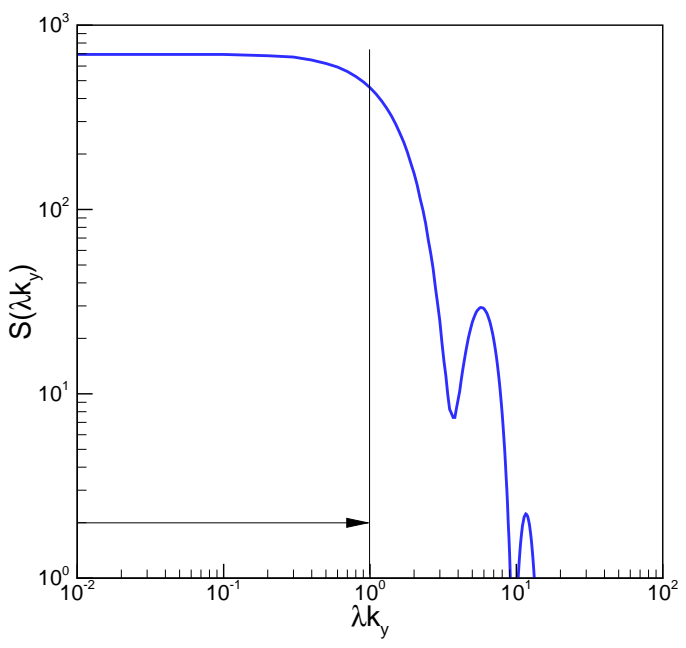

(d)

Figure 5. (a) Streamwise, (b) spanwise surface pressure spectra and their universal spectra (c) and (d) respectively. 


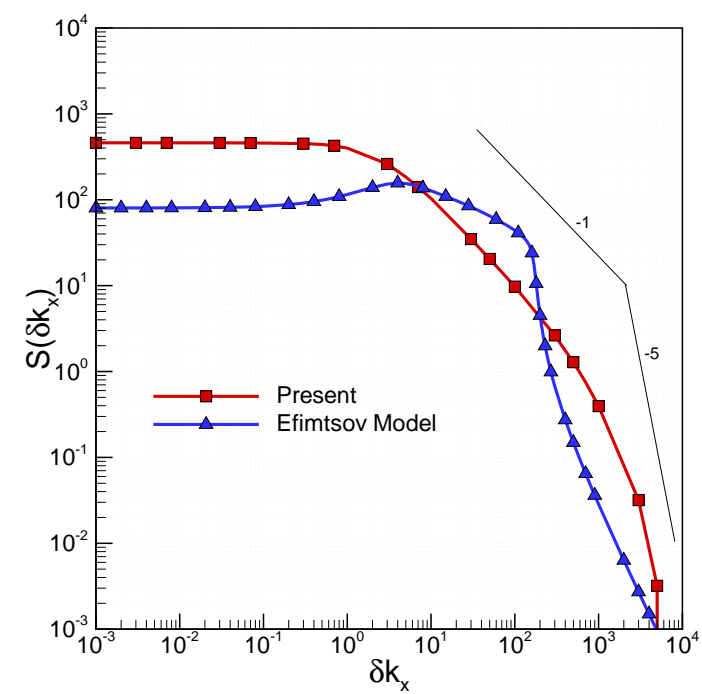

(a)

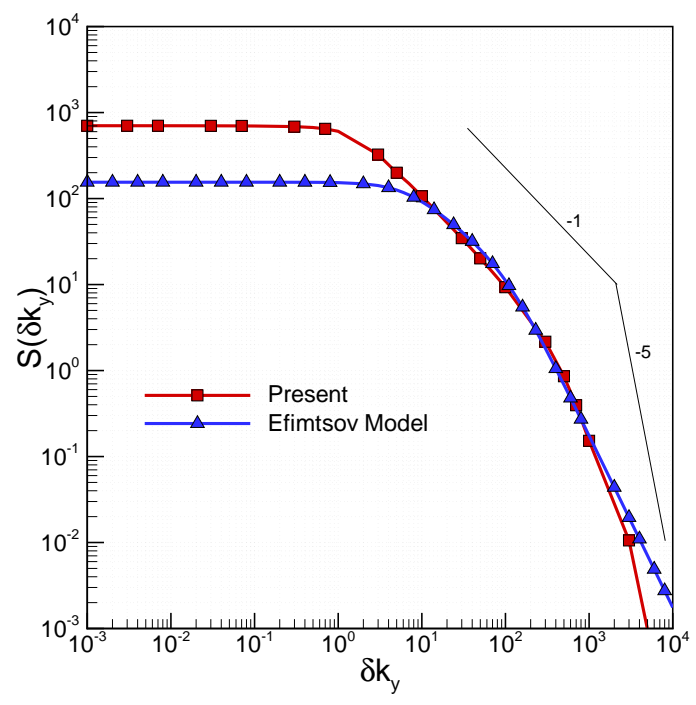

(b)

Figure 6. (a) Streamwise and (b) spanwise wavenumber spectra.

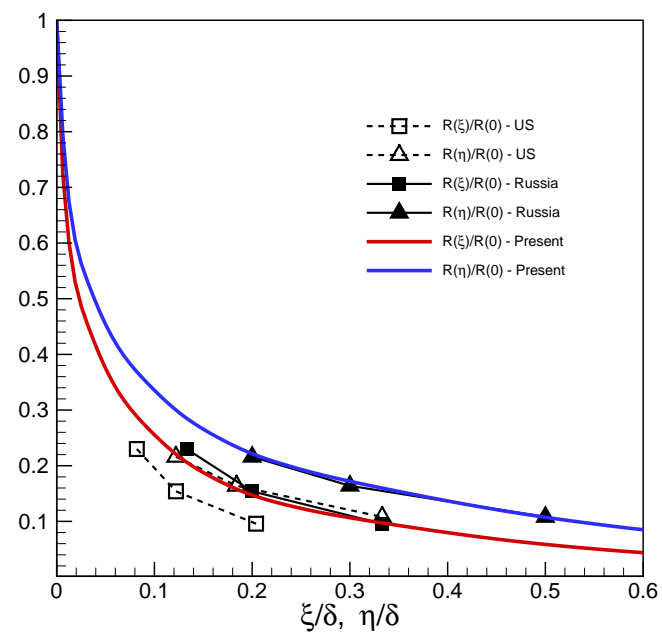

(a)

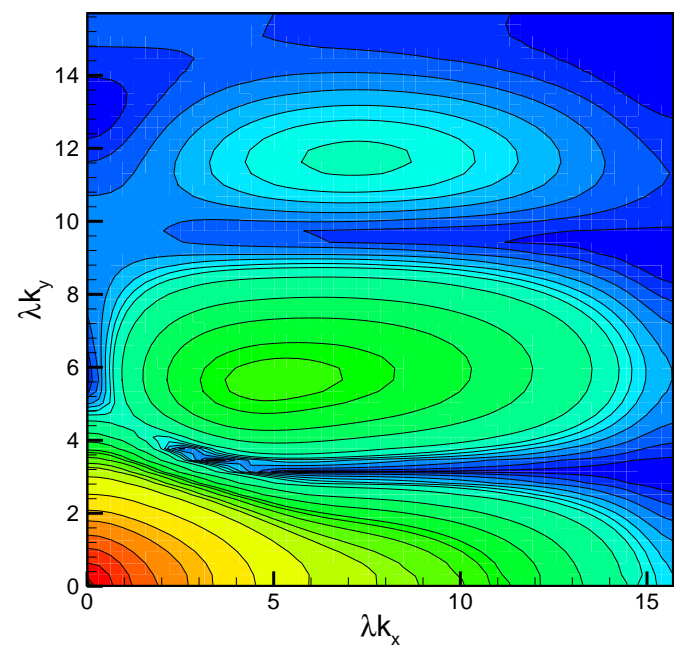

(b)

Figure 7. (a) Spatial correlations and (b) Stream-spanwise universal spectrum.

12 of 12

American Institute of Aeronautics and Astronautics 